\section{Molecular and circadian controls of ameloblasts}

\author{
Athanassiou-Papaefthymiou M, Kim D, Harbron L, Papagerakis S, Schnell S, Harada \\ H, Papagerakis P. Molecular and circadian controls of ameloblasts. \\ Eur J Oral Sci 2011; 119 (Suppl. 1): 35-40. (C) 2011 Eur J Oral Sci
}

Stage-specific expression of ameloblast-specific genes is controlled by differential expression of transcription factors. In addition, ameloblasts follow daily rhythms in their main activities (i.e. enamel protein secretion and enamel mineralization). This time-related control is orchestrated by oscillations of clock proteins involved in the regulation of circadian rhythms. Our aim was to identify the potential links between daily rhythms and developmental controls of ameloblast differentiation. The effects of the transcription factors distal-less homeobox $3(D l \times 3)$ and runt-related transcription factor 2 (Run $x$ ), and the clock gene nuclear receptor subfamily 1 , group D, member 1 ( Nr 1d1), on secretory and maturation ameloblasts [using stage-specific markers amelogenin (Amelx), enamelin (Enam), and kallikrein-related peptidase 4 (Klk4)] were evaluated in the HAT-7 ameloblast cell line. Amelx and Enam steady-state mRNA expression levels were down-regulated in Runx2 over-expressing cells and up-regulated in $D l \times 3$ over-expressing cells. In contrast, $K l k 4$ mRNA was up-regulated by both $D l \times 3$ and Runx2. Furthermore, a temporal and spatial relationship between clock genes and ameloblast differentiation markers was detected. Of interest, clock genes not only affected rhythmic expression of ameloblast-specific genes but also influenced the expression of Runx2. Multiscale mathematical modeling is being explored to further understand the temporal and developmental controls of ameloblast differentiation. Our study provides novel insights into the regulatory mechanisms sustaining ameloblast differentiation.

\section{Maria Athanassiou- Papaefthymiou ${ }^{1,2 *}$, Doohak Kim ${ }^{1 *}$, Lindsay Harbron ${ }^{1 *}$, Silvana Papagerakis ${ }^{2}$, Santiago Schnell ${ }^{3}$, Hidemitsu Harada ${ }^{4}$, Petros Papagerakis $^{1}$}

${ }^{1}$ Department of Orthodontics and Pediatric Medicine, Center for Computational Medicine and Bioinformatics, Center for Organogenesis, University of Michigan Schools of Dentistry and Medicine, Ann Arbor, MI, USA; ' 2 Laboratory for Metastasis of Head and Neck Cancer, the

Kresge Hearing Research Institute, Department of Otolaryngology and Comprehensive Cancer Research Center, the University of Michigan Medical School, Ann Arbor, MI, USA; ${ }^{3}$ Department of Molecular and Integrative Physiology, Brehm Center for Diabetes Research, the University of Michigan Medical School, Ann Arbor, MI, USA; ${ }^{4}$ Division of Histology and Developmental Biology, Department of Oral Biology, School of Dentistry, Iwate Medical University, Morioka, Iwate, Japan

*Authors who contributed equally to this work.

Petros Papagerakis, Department of Orthodontics \& Pediatric Dentistry, School of Dentistry, University of Michigan, $1011 \mathrm{~N}$. University, Ann Arbor, MI 48109, USA

Telefax: +1-734-7638100

E-mail: petrosp@umich.edu

Key words: ameloblast gene regulation; circadian rhythms; clock genes; enamel; multiscale modeling

Accepted for publication October 2011
Dental enamel is formed mainly during two distinct developmental stages $(1,2)$ : the secretory stage (at the end of which the full thickness of enamel is completed), and the maturation stage (during which residual organic material is removed and the tissue is eventually occluded by hydroxyapatite crystals). Ameloblasts, the cells responsible for making enamel, are specialized epithelial cells with distinct morphological features that change during ameloblast differentiation (2). Secretory and maturation ameloblasts are characterized by restricted expression of enamel stage-specific genes and by stagespecific functions (3-8). However, the control of gene expression in ameloblasts, resulting in specialized functions that direct enamel secretion and maturation, is unclear (9-13).

Mineralized tissue development results from a complex temporo-spatial expression of adhesion molecules and growth and transcription factors. Different mineralized tissues share common signaling pathways. Runt-related transcription factor 2 (Runx2) and distal-less homeobox $3(D l \times 3)$ are both key regulatory transcription factors that control bone formation (14). Run $x 2$ is also expressed by maturation-stage ameloblasts during enamel formation and Runx2 mutations result in enamel abnormalities (15). Similarly to Runx2, Dlx3 is strongly expressed in ameloblasts (16) and Dlx3 mutations are linked to amelogenesis imperfecta (AI) (17). This study aimed to elucidate the transcriptional targets of Runx2 and Dlx3 during amelogenesis.

In addition to the stage-specific regulation by transcription factors, it has long been suggested that gene expression and dental tissue formation are under circadian control both in rodents and in humans. Previous studies demonstrated that the formation of incremental lines in rat dentin reflect a circadian rhythm in the synthesis and secretion of collagen (18). Similarly to rat dentin, human enamel is formed by appositional growth, leaving growth marks on the enamel surface every $24 \mathrm{~h}$ during the secretory stage (19). In addition, the secretion of amelogenin (AMELX) clearly shows daily oscillations (20). At a later stage of development - the maturation stage - ameloblasts oscillate between smooth-ended and 
ruffle-ended morphologies every $8 \mathrm{~h}$ in rat and express a different set of proteins at each part of their cycle (2). Therefore, ameloblast differentiation is directly correlated with cyclical gene expression and specialized cell functions. However, no direct evidence for a 'dental' circadian clock exists. It is also unclear if genes expressed in ameloblasts are under circadian control and how circadian control affects ameloblast differentiation and enamel formation. This is the first study that aims to elucidate how clock genes regulate formation and maturation of mineralized tissues and how stage-specific regulation is linked to daily circadian controls.

\section{Material and methods}

\section{Cell culture and study of circadian effects}

The ameloblast-like cell line, HAT-7 (21), was cultured in Dulbecco's modified Eagle's minimum essential medium (DMEM)/F12 (1:1, volume by volume) containing L-glutamine, $15 \mathrm{mM}$ HEPES, and 10\% fetal bovine serum (Invitrogen, Carlsbad, CA, USA). Cells were passaged just before confluence and plated in six-well plates. For measuring the circadian effects of clock genes, HAT-7 cells were allowed to reach $80 \%$ confluence and then the medium was supplemented with $0.1 \mathrm{mM}$ forskolin. Forskolin is known to induce cell cycle synchronization of cultured cells (22). Total RNA was harvested every $4 \mathrm{~h}$ for $28 \mathrm{~h}$ using TRIzol (Invitrogen). Two micrograms of RNA were reverse transcribed with TaqMan reverse transcription reagents (Applied Biosystems, Branchbury, NJ, USA), following the manufacturer's recommendations. cDNA was then quantified and used for real-time quantitative RT-PCR (qRT-PCR). qRT-PCR was conducted using SYBR Green (Invitrogen) and specific primers (Table 1) for beta-actin $(A c t b)$, Amelx, enamelin (Enam), ameloblastin (Ambn), matrix metalloproteinase $20(\mathrm{Mmp} 20)$, kallikrein-related peptidase 4 (Klk4), aryl hydrocarbon receptor nuclear translocator-like $(B m l \alpha 1)$, nuclear receptor subfamily 1, group D, member 1 (Nr1d1), Dlx3, and Runx2.

\section{Transfection and real-time qRT-PCR}

HAT-7 cells were cultured in DMEM/F12 (1:1, volume by volume) containing L-glutamine, $15 \mathrm{mM}$ HEPES, and 10\% fetal bovine serum (Invitrogen), then passaged and plated in six-well plates. The cells were then transfected at $80 \%$ confluence with $2 \mathrm{mg}$ of Nr1d1 or Dlx3 (gifts of Dr. Maria M. Morasso, Developmental Skin Biology Section, NIAMS-NIH, DC, USA) or Runx2 (a gift of Dr. Renny Franceschi, University of Michigan, Ann Arbor, MI, USA) or control (empty pCDNA) expression vectors using Lipofectamine LTX and Plus Reagent (Invitrogen). Total RNA was isolated 24 or $48 \mathrm{~h}$ later from HAT-7 cells using TRIzol (Invitrogen), and $2 \mu \mathrm{g}$ of RNA was reversely transcribed with following the manufacturer's recommendations (Applied Biosystems). The resulting cDNA was then amplified by qRT-PCR. RT-PCR amplifications were performed at $95^{\circ} \mathrm{C}$ for $30 \mathrm{~s}$, at $60^{\circ} \mathrm{C}$ for $30 \mathrm{~s}$, and at $72^{\circ} \mathrm{C}$ for $30 \mathrm{~s}$ using specific primers (Table 1). The relative expression levels for each gene were calculated based on the expression levels of $A c t b$ and the differences are presented in graphs using the $2^{-\Delta \Delta C_{\mathrm{T}}}$ method. $P$-values were calculated using two-sample $t$-test. RT-PCR products were also subcloned
Table 1

RT-PCR primers

\begin{tabular}{|c|c|}
\hline Gene name & Sequence $5^{\prime}-3^{\prime}$ \\
\hline \multirow[t]{2}{*}{$A m b n$} & Forward: GTCCAGAAGGCTCTCCACTG \\
\hline & Reverse: GTCATTGGGGAAAGCAAGAA \\
\hline \multirow[t]{2}{*}{ Amelx } & Forward: TACCACCTCATCCTGGAAGC \\
\hline & Reverse: CTGTTGAGACAGCACAGGGA \\
\hline \multirow[t]{2}{*}{ Dlx3 } & Forward: ACCCAGTGTCGGTGAAAGAG \\
\hline & Reverse: GCCAGATACTGGGCTTTCTG \\
\hline \multirow[t]{2}{*}{ Enam } & Forward: GATGCCCATGTGGCCTCCACCA \\
\hline & Reverse: GCCAAATGGTGGGAATGGCTGA \\
\hline \multirow[t]{2}{*}{ Klk4 } & Forward: ACAAGGGCTCGTGTCTATGG \\
\hline & Reverse: GTCTCAGGTTCCCTCAGCAG \\
\hline \multirow[t]{2}{*}{ Mmp20 } & Forward: AGCTCGTCCTTTGATGCAGT \\
\hline & Reverse: TGGACATTAGCTGGGGAAAG \\
\hline \multirow[t]{2}{*}{$N r 1 d 1$} & Forward: CTTCCGTGACCTTTCTCAGCA \\
\hline & Reverse: TGTGCGGCTCAGGAACATCAC \\
\hline \multirow[t]{2}{*}{ Runx 2} & Forward: CCGTCCATCCACTCTACC \\
\hline & Reverse: TGCCTGGCTCTTCTTACTG \\
\hline \multirow[t]{2}{*}{ Actb } & Forward: AAGTACCCCATTGAACACGG \\
\hline & Reverse: ATCACAATGCCAGTGGTACG \\
\hline \multirow[t]{2}{*}{$B m l \alpha 1$} & Forward: CCAAGAAAGTATGGACACAGACAAA \\
\hline & Reverse: GCATTCTTGATCCTTCCTTGGT \\
\hline
\end{tabular}

Detailed sequence information is provided here for all the primers used in this study. The following gene symbols are used in this table: beta-actin $(A c t b)$, amelogenin (Amelx), enamelin (Enam), ameloblastin (Ambn), matrix metalloproteinase 20 (Mmp20), kallikrein-related peptidase 4 (Klk4), aryl hydrocarbon receptor nuclear translocator-like $(B m l \alpha 1)$, nuclear receptor subfamily 1 , group D, member 1 ( $N r 1 d 1)$, distal-less homeobox 3 (Dlx3), and runt-related transcription factor 2 (Runx2).

into the pGEM-T Easy vector (Promega, Madison, WI, USA) and mRNA expression was confirmed by direct sequencing.

\section{Results \\ Effects of Runx2 and DIx3 on ameloblast-specific gene expression}

HAT-7 cells were transfected with expression vector containing Runx2 and the changes in mRNA expression levels for stage-specific ameloblast genes (i.e. Amelx, Enam, and Klk4) were evaluated by qRT-PCR. Our data showed that Runx2 down-regulates Enam mRNA levels (Fig. 1A) and Amelx mRNA levels (data not shown) and up-regulates Klk4 mRNA levels (Fig. 1A). HAT-7 cells were also transfected with the expression vector containing Dlx3. The ameloblast-specific mRNA Amelx and Enam (markers of secretory ameloblasts) and Klk4 (a marker of maturation-stage ameloblasts) were all up-regulated upon the over-expression of Dlx3 in HAT-7 cells (Fig. 1B). Cells transfected with a control vector (pCDNA) showed no significant changes.

\section{Effects of Nr1d1 on ameloblast-specific gene expression}

Cell cycle synchronized HAT-7 cells were used to evaluate the levels of expression of clock genes in ameloblasts at regular daily intervals. Several clock genes were 

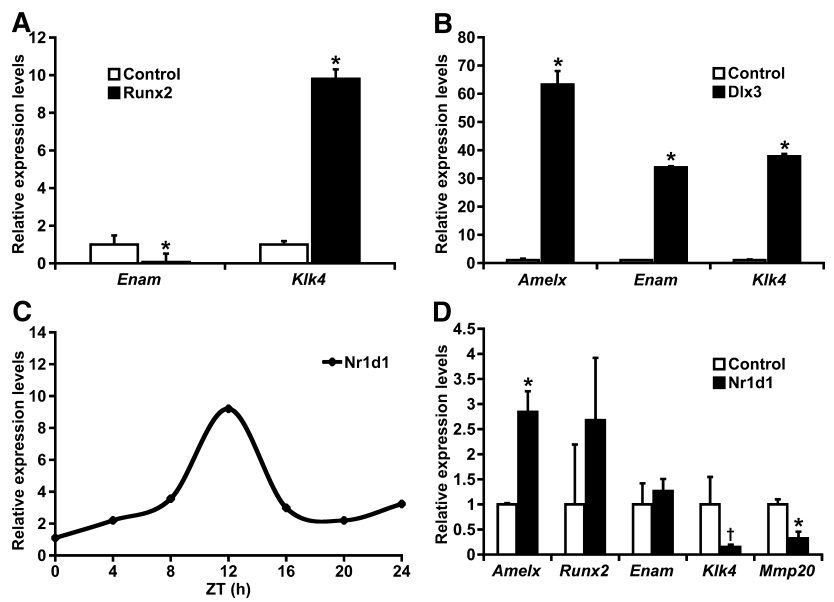

Fig. 1. (A) Effects of runt-related transcription factor 2 (Runx2) on HAT-7 ameloblasts. Over-expression of Runx 2 resulted in the down-regulation of enamelin (Enam) $(P<0.05)$ and in the up-regulation of kallikrein-related peptidase $4(K l k 4)(P<0.05)$. Experiments were performed in triplicate. (B) Effects of distal-less homeobox 3 (Dlx3) on HAT-7 ameloblasts. Over-expression of Dlx3 encoding full-length protein (1-287 amino acids) resulted in the up-regulation of amelogenin (Amelx), Enam, and Klk4 $(P<0.05$ for all three genes). All data were evaluated $24 \mathrm{~h}$ after transfection. (C) Circadian oscillations at the RNA level were found for clock genes in ameloblasts after cell cycle synchronization using forskolin. The results are shown here for nuclear receptor subfamily 1 , group D, member 1 ( Nrldl), which showed a surge in expression $12 \mathrm{~h}$ after cell cycle synchronization ZT, Zeitgeist. (D) Transfection of HAT-7 cells with Nrldl resulted in statistically significant up-regulation of Amelx $(P<0.05)$ and down-regulation of Klk4 $(P<0.056)$ and Mmp20 $(P<0.05)$. In addition, the levels of Runx2 mRNA were also up-regulated $24 \mathrm{~h}$ after transfection of HAT-7 cells with Nr1d1 (not statistically significant). In contrast, steady-state mRNA levels of Enam remained unchanged upon the over-expression of $\mathrm{Nr} 1 d 1$. *Statistically significant changes; $†$ Very close to being statistically significant changes.

detected and found to oscillate at the RNA level. One of the most regularly oscillated clock mRNA in ameloblasts was Nrldl (Fig. 1C). We then decided to evaluate if over-expression of Nrldl changes the expression of mRNA levels of ameloblast-specific genes. Over-expression of Nrldl resulted in the up-regulation of Amelx and in the down-regulation of Mmp20 and Klk4 mRNAs (Fig. 1D). In contrast, the expression of Enam (Fig. 1D) and Ambn (not shown) mRNAs was unchanged upon the over-expression of Nrld1. Furthermore, we also evaluated changes in the levels of expression of Runx2, a key regulator of ameloblast-specific genes (Fig. 1D).

\section{Multilevel and time-dependent control of ameloblast- specific gene expression and cell functions}

We are also analyzing how the stage-specific regulation and circadian control networks govern ameloblast differentiation and enamel formation using a multiscale modeling approach. In our computational approach, cells are modeled as discrete entities that respond to intracellular and extracellular signals, which are modeled continuously with differential equations. Key circadian clock genes involved in amelogenesis are being integrated into a Boolean gene network. In their simplest form, Boolean models are interaction networks where each biochemical species is represented as a node in one of two possible states: expressed ('on' or 1) or non-expressed ('off' or 0) (23). Transfer functions between states are derived from biochemical interactions using logical operators (e.g. AND, OR, and NOT). The response to signals from the intracellular gene network determines whether each cell differentiates, proliferates or dies, and therefore directly influences the cellular and the extracellular tissue scales. The spatial distribution of cells is computed using a continuous macroscopic tissue model based on the viscous liquid theory of tissue dynamics. Finally, the number and spatial configuration of cells are used to activate tissue signals, which in turn were input into the Boolean model (Fig. 2). This combination of discrete and continuous modeling of several steps of amelogenesis will be used to analyze key cellular events (such as ameloblast extension of differentiation) and to predict the most important regulatory networks necessary for enamel formation. This multiscale modeling approach provides a powerful tool for addressing questions of how cells interact with each other and their environment, and how these interactions, in turn, affect gene expression.

\section{Discussion}

Our laboratory focuses on the study of gene expression during ameloblast differentiation. Enamel formation depends largely on a complex temporo-spatial expression of adhesion molecules and growth and transcription factors, described in early tooth development (24), which continues during cell differentiation and enamel formation. During the first stage of amelogenesis, secretory ameloblasts delineate the enamel space, and Amelx, Enam, and Ambn proteins are secreted and assembled to form an extracellular framework (25). Ameloblasts then transport calcium and phosphate ions into this framework, forming hydroxyapatite crystallites (4). During enamel formation, the organic materials in the matrix are 




Fig. 2. A multiscale Boolean model is being designed to predict the complex interactions between circadian controls and stagespecific regulators such as runt-related transcription factor 2 (Runx2) and distal-less homeobox 3 (Dlx3) that control gene expression in ameloblasts and ultimately orchestrate ameloblast differentiation and enamel formation. A schematic view is presented of the multiscale nature of our model composed of four different levels. At the genetic level we integrate the main genes involved in the regulation of amelogenesis within a Boolean network, which results in regulatory signals that control differentiation. The response to these signals occurs at the cellular level, determining whether each cell progresses through differentiation or dies. Given this information, at the macroscopic model the new spatial distribution of the cells is computed at the tissue level. The number and spatial configuration of cells determine the activation of the regulatory signals, which in turn input to the genetic level. Clock genes induce daily oscillations of key transcription factors, which, in the model, activate stage-specific ameloblast genes at the genetic level.

degraded by two proteases - Mmp20 and Klk4 - leaving behind a fluid-filled porous tissue where secondary crystal growth and mineral accretion can occur to go on to produce the final mature enamel. Defects in the formation of enamel are seen in patients with AI, and mutations in AMELX, ENAM, KLK4, and MMP20 have been implicated in the etiology of AI (26). Of all these genes, expression of ENAM is exclusive to secretory ameloblasts (27). Amelx and Mmp20 are expressed in secretory ameloblasts as well as in odontoblasts (5), whereas Klk4 is mainly expressed in the maturation-stage ameloblasts (28).

The aim of this study was to test the hypothesis that Runx 2 and $D l \times 3$ are involved in ameloblast stage-specific gene regulation. We found that Runx2 down-regulates Enam and up-regulates Klk4. This is consistent with the developmental expression patterns of Enam and Klk4. The expression of Runx2 is initiated at the end of the secretory stage when Enam expression is suppressed. Runx 2 expression continues during the maturation stage when Klk4 is exclusively expressed. Therefore, we propose that Runx2 is a key regulator of ameloblast differentiation with a role which involves suppressing genes expressed in the secretory stage, such as Amelx and Enam and up-regulating genes of the maturation stage, such as Klk4. Further in vivo studies are needed to confirm these preliminary indications and to identify any partners of Runx2 that may be involved in the down-regulation or up-regulation of ameloblast genes studied here.

Dlx3 is another major player in amelogenesis. Dlx3 is strongly expressed by ameloblasts (16) and Dlx3 mutations result in AI (17). In this study, we showed that Dlx3 up-regulates the expression of Amelx, Enam, and Klk4. These findings are consistent with suggested roles of Dlx 3 during both the secretory and the maturation stages of amelogenesis. Our data are also in accordance with previous studies that reported Amelx regulation by $D l \times 2$ based on gel-shift assays and promoter Dlx2-binding site predictions (29). It is possible that synergistic and/or competitive relationships between DLX proteins take place during amelogenesis. More studies are needed to clarify the precise roles of the $D l x$ family of transcription factors in amelogenesis. Nevertheless, our data support a key role of Dlx3 in ameloblast differentiation.

Circadian rhythms are self-sustained endogenous oscillations that occur over a $24-\mathrm{h}$ period. They correspond to the environmental light-dark cycles of an organism but persist even after the light-dark stimulus has been removed. These biological rhythms are involved in most physiological processes. Although there is a site in the suprachiasmatic nucleus of the brain that is considered as the 'master clock', peripheral clocks have been found in several tissues in the body. The relationship between these two types of circadian biological clocks is, as yet, unclear (30). Several genes have been identified as core maintainers of the circadian rhythm. The main mammalian genes include Circadian Locomotor Output Cycles Kaput (Clock), Brain and Muscle Aryl Hydrocarbon Receptor Nuclear Translocation (ARNT)-like (Bmall), Period 1 (Per 1$)$, Period 2 (Per2), Period 3 (Per3), Cryptochromes (Cryl) and Cry2. The genes Nrld1, Nr1d2, RAR-Related Orphan Receptor Alpha (Ror $\alpha$ ), and Albumin D-binding protein ( $D b p$ ) also play a key role in modifying the expression of the main clock genes (31). Transcription of these 'clock genes' oscillates over a 24-h period and their output signals induce rhythms of target gene expression that create patterns in physiological processes. Inducing a rhythm involves the binding of a clock gene transcription factor to the promoter region of a clock-controlled gene (32).

We have recently shown that clock genes and clock proteins are expressed during ameloblast differentiation $(20,33)$. This present study further supports the concept that clock genes are expressed in ameloblasts and that their expression oscillates during 24-h intervals. Furthermore, we presented evidence showing that clock genes regulate several ameloblast stage-specific genes, supporting the idea that clock genes are key regulators of ameloblast differentiation. These data are consistent with our previous discoveries reporting that the amounts of AMELX secreted vary during different daily intervals (20). In addition, we showed that the over-expression of Nrldl results in the up-regulation of Runx 2 mRNA, a key transcription factor strongly expressed in maturation ameloblasts. Our data also showed that Runx2 regulates the expression of Enam and Klk4 mRNAs. We therefore hypothesize that clock genes may regulate the daily 


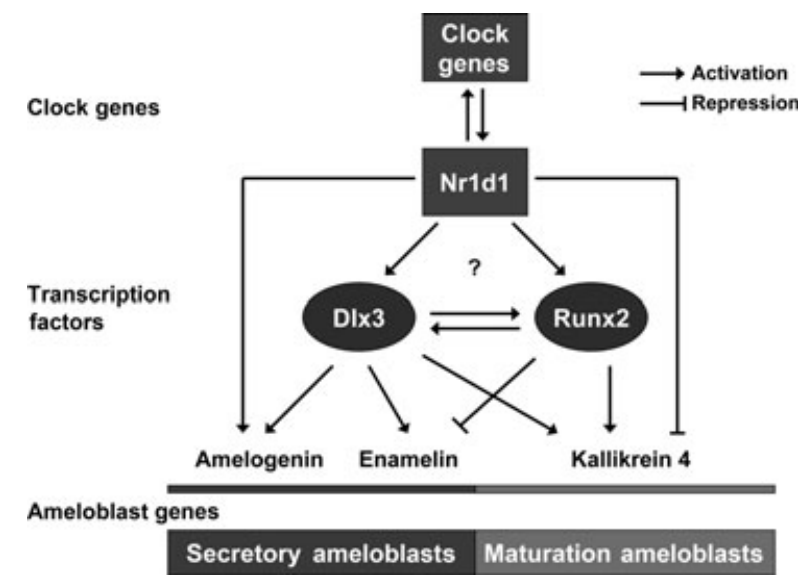

Fig. 3. This cartoon summarizes our findings reported here. Gene regulation in ameloblasts is orchestrated by stage-specific controls, as shown for amelogenin (Amelx), enamelin (Enam), and kallikrein-related peptidase 4 (Klk4) regulation by runtrelated transcription factor 2 (Runx2) and distal-less homeobox 3 (Dlx3). Gene expression in ameloblasts is also subject to circadian controls, as shown for Amelx, matrix metalloproteinase 20 (Mmp20), Klk4, and Runx2 regulation by nuclear receptor subfamily 1 , group D, member 1 ( $N r l d 1)$. Accordingly, we postulate that circadian control of ameloblast genes can be direct but also indirect (e.g. through Runx2). It is currently unknown if $D l \times 3$ is also subject to circadian regulation. It is also unknown if Runx2 and/or Dlx3 can regulate the expression of clock genes in ameloblasts in a negative-feedback loop. Another complexity is that clock genes regulate each other, resulting in complex network interactions. We are currently analyzing these networks and their effects on ameloblast differentiation and enamel formation using mathematical modeling.

variations of gene expression in ameloblasts either directly, by regulating their transcriptional rates, or indirectly, by regulating the expression of key transcription factors (Runx2 in our case) that regulate the expression of ameloblast genes. More studies are needed to understand the precise roles of clock genes in enamel formation. Nevertheless, we suggest that in addition to the stage-specific controls, amelogenesis is subject to very precise, rhythmic daily controls of gene-expression levels and cell activity (Fig. 3).

In conclusion, our study offers novel insights into the role of clock genes in ameloblast differentiation and explores the potential links between circadian control and stage-specific regulation of ameloblast genes. Our hypothesis, that an ameloblast peripheral clock regulates enamel formation orchestrating the expression of ameloblast-specific genes, is further strengthened. Direct links between changes in the expression of clock genes and dental diseases remain to be confirmed using in vivo models. Nevertheless, this initial study, using an ameloblast cell line, lays the foundation for more research in the chronobiology of tooth development and diseases.

Acknowledgements - This work was supported by NIH grant DE018878-01A1 (to P.P.) and by funds from the UM Department of Orthodontics and Pediatric Dentistry. We also thank Drs Hu, Simmer and Yamakoshi and their laboratory personnel at the University of Michigan for helpful discussions.
Conflicts of interest - The authors declare no conflicts of interest.

\section{References}

1. Smith CE, Nanci A. Secretory activity as a function of the development and maturation of ameloblasts. Connect Tissue Res 1989; 22: 147-156.

2. Sмith CE. Cellular and chemical events during enamel maturation. Crit Rev Oral Biol Med 1998; 9: 128-161.

3. Uchida T, Tanabe T, Fukae M, Shimizu M. Immunocytochemical and immunochemical detection of a $32 \mathrm{kDa}$ non amelogenin and related proteins in porcine tooth germs. Arch Histol Cytol 1991; 54: 527-538.

4. Simmer JP, Fincham AG. Molecular mechanisms of dental enamel formation. Crit Rev Oral Biol Med 1995; 6: 84-108.

5. Papagerakis P, Macdougall M, Hotton D, BailleulForestier I, Oboeuf M, Berdal A. Expression of amelogenin in odontoblasts. Bone 2003; 32: 228-240.

6. Moffatt P, Smith CE, Sooknanan R, St-Arnaud R, Nanci A. Identification of secreted and membrane proteins in the rat incisor enamel organ using a signal-trap screening approach. Eur J Oral Sci 2006; 114: 139-146.

7. Moffatt P, Smith CE, St-Arnaud R, Nanci A. Characterization of Apin, a secreted protein highly expressed in toothassociated epithelia. J Cell Biochem 2008; 103: 941-956.

8. Hu JC, Hu Y, Smith CE, McKee MD, Wright JT, Yamakoshi Y, Papagerakis P, Hunter GK, Feng JQ, YAMAKoshi F, Simmer JP. Enamel defects and ameloblastspecific expression in enamelin knockout/LacZ knockin mice. $J$ Biol Chem 2008; 283: 10858-10871.

9. Gibson CW. Regulation of amelogenin gene expression. Crit Rev Eukaryot Gene Expr 1999; 9: 45-57.

10. Papagerakis P, Hotton D, Lezot F, Brookes S, Bonass W, Robinson C, Forest N, BERdAl A. Evidence for regulation of amelogenin gene expression by 1,25-dihydroxyvitamin $\mathrm{D}(3)$ in vivo. J Cell Biochem 1999; 76: 194-205.

11. Hu YY, Papagerakis P, Ye L, Feng JQ, Simmer JP, Hu JC-C. Distal cis-regulatory elements are required for tissue-specific expression of Enamelin (Enam). Eur JOral Sci 2008; 116: 113-123.

12. Papagerakis P, Hu Y, Ye L, Feng JQ, Simmer JP, Hu JC. Identifying promoter elements necessary for enamelin tissuespecific expression. Cells Tissue Organs 2009; 189: 98-104.

13. Bei M, Stowell S, MaAs R. Msx2 controls ameloblast terminal differentiation. Dev Dyn 2004; 231: 758-765.

14. Hassan MQ, Javed A, Morasso Mi, Karlin J, Montecino M, Van WiJnen AJ, Stein GS, Stein JL, Lian JB. Dlx3 transcriptional regulation of osteoblast differentiation: temporal recruitment of Msx2, Dlx3, and Dlx5 homeodomain proteins to chromatin of the osteocalcin gene. Mol Cell Biol 2004; 24: 9248-9261.

15. D'souza Rn, Aberg T, Gaikwad J, Cavender A, Owen M, Karsenty G, TheslefF I. Cbfal is required for epithelialmesenchymal interactions regulating tooth development in mice. Development 1999; 126: 2911-2920.

16. Ghoul-Mazgar S, Hotton D, Lezot F, Blin-Wakkach C, Asselin A, Sautier JM, Berdal A. Expression pattern of Dlx3 during cell differentiation in mineralized tissues. Bone 2005; 37: 799-809.

17. Price JA, Bowden DW, Wright JT, Pettenati MJ, Hart TC. Identification of a mutation in Dlx 3 associated with trichodento-osseous (TDO) syndrome. Hum Mol Genet 1998; 7: $563-$ 569.

18. Ohtsuka M, Saeki S, Igarashi K, Shinoda H. Circadian rhythms in the incorporation and secretion of $3 \mathrm{H}$-proline by odontoblasts in relation to incremental lines in rat dentin. J Dent Res 1998; 77: 1889-1895.

19. RISNES S. Enamel apposition rate and the prism periodicity in human teeth. Scand J Dent Res 1986; 94: 394-404.

20. Simmer JP, Papagerakis P, Smith CE, Fisher DC, Rountrey AN, Zheng L, Hu JC-C. Regulation of Dental Enamel Shape and Hardness. J Dent Res 2010; 89: 1024-1038. 
21. Kawano S, Morotomi T, Toyono T, Nakamura N, Uchida T, Ohishi M, Toyoshima K, Harada H. Establishment of dental epithelial cell line (HAT-7) and the cell differentiation dependent on Notch signaling pathway. Connect Tissue Res 2002; 43: 409-412.

22. Atanasov AG, Leiser D, Roesselet C, Noti M, Corazza N, SChoOnJAns K, BRUnNer T. Cell cycle-dependent regulation of extra-adrenal glucocorticoid synthesis in murine intestinal epithelial cells. FASEB J 2008; 22: 4117-4125.

23. Ribba B, Colin T, Schnell S. A multi-scale mathematical model of cancer, and its use in analyzing irradiation therapies. Theor Biol Med Model 2006; 3: 1-19.

24. Jernvall J, TheslefF I. Reiterative signaling and patterning during mammalian tooth morphogenesis. Mech Dev 2000; 92: 19-29.

25. Fincham AG, Moradianoldak J, Simmer JP. The structural biology of the developing dental enamel matrix. J Struct Biol 1999; 126: 270-299.

26. Papagerakis P, Lin HK, Lee Ky, Hu Y, Simmer JP, Bartlett JD, Hu JC. Premature stop codon in MMP20 causing amelogenesis imperfecta. J Dent Res 2008; 87: 5659 .
27. Hu JC, Sun X, Zhang C, Simmer JP. A comparison of enamelin and amelogenin expression in developing mouse molars. Eur J Oral Sci 2001; 109: 125-132.

28. Lu Y, Papagerakis P, Yamakoshi Y, Hu J, Bartlett J, SiMMER JP. Functions of KLK4 and MMP-20 in dental enamel formation. Biol Chem 2008; 389: 695-700.

29. Lezot F, Thomas B, Greene S, Hotton D, Yuan ZA, Castaneda B, Bolan A, Depew M, Sharpe P, Gibson C, BERDAL A. Physiological Implications of DLX Homeoproteins in Enamel Formation. J Cell Physiol 2008; 216: 688-697.

30. Brown SA, Schibler U. The ins and outs of circadian timekeeping. Curr Opin Genet Dev 1999; 9: 588-594.

31. Ukai-Tadenuma M, Kasukawa T, Ueda HR. Proof-by-synthesis of the transcriptional logic of mammalian circadian clocks. Nat Cell Biol 2008; 10: 1154-1163.

32. Jin X, Shearman LP, Weaver DR, Zylka MJ, De Vries GJ, REPPERT SM. A molecular mechanism regulating rhythmic output from the suprachiasmatic circadian clock. Cell 1999; 96: 57-68.

33. Zheng L, Papagerakis S, Schnell S, Hoogerwerf WA, PaPAGERAKIs P. Expression of Clock Proteins in Developing Tooth. Gene Expr Pat 2011; 11: 202-206. 